\title{
RADIATION HEAT TRANSFER WITH ABLATION IN A FINITE PLATE
}

F. A. A. Gomes ${ }^{\text {, }}$
J. B. C. Silva
and A. J. Diniz

\section{NOMENCLATURE}

$A, B$ heat flux constants

$C, D$ heat flux constants

$c^{*} \quad$ heat capacity per unit volume

$c_{p}^{*} \quad$ specific heat

$E_{2}^{p} \quad$ radiant energy

$E, F, G$ constants defined in the analytical solution by the GITT

$H^{*} \quad$ heat of ablation

$K^{*} \quad$ thermal conductivity

$\left(\bar{K}^{*}\right)^{-1} \quad$ characteristic length

$L^{*} \quad$ thickness of the slab

$N \quad$ total number of elemental

$Q \quad$ dimensionless heat flux, $\frac{q_{e}^{*}}{\lambda^{*} T_{0} \bar{K}^{*}}$

$q_{e}^{*} \quad$ dimensional wall heat flux

$T^{*} \quad$ dimensionless temperature

$T_{0}^{*} \quad$ initial temperature

$T_{m}{ }^{*} \quad$ melting temperature

$t^{*} \quad$ dimensional time

$t_{c}^{*} \quad$ characteristic time, $\bar{K}^{* 2} / \alpha^{*}$

$t_{m}{ }^{*} \quad$ time at the instant when melting starts

$t_{r}^{*} \quad$ reference time

$v \quad$ ablation speed, $d \eta_{b}(\tau) / d \tau \equiv d S(t) / d t$
$X \quad$ dimensionless coordinate, $\bar{K}^{*} x^{*}$

$X^{*} \quad$ dimensional coordinate

\section{Greek symbols}

$\alpha \quad$ absoptivity

$\alpha^{*} \quad$ thermal diffusivity

$\varepsilon \quad$ emissivity

$\phi_{i} \quad$ eigenfunction of the ablation period

$\eta \quad$ modified auxiliary coordinate, $\eta=1-x$

$\eta_{b} \quad$ width of ablative material

$\lambda^{*} \quad$ wavelength which energy is emitted

$\lambda_{i} \quad$ eigenvalue of the ablation period

$\mu_{i} \quad$ eigenvalue of the preablation period

$v^{*} \quad$ inverse of Stefan number, $\frac{H^{*}}{c^{*}\left(T_{m}^{*}-T_{0}^{*}\right)}$

$\theta \quad$ dimensionless temperature, $\frac{\left(T^{*}-T_{0}^{*}\right)}{\left(T_{m}^{*}-T_{0}^{*}\right)}$

$\rho^{*} \quad$ specific density

$\tau \quad$ dimensionless time, $t^{*} / t_{c}^{*}$

$\tau_{r} \quad$ dimensionless reference time, $t_{r}^{*} / t_{c}^{*}$

$\tau_{m} \quad$ dimensionless time when melting just starts, $t_{m}^{*} / t_{c}^{*}$ 


\section{CIÊNCIA/SCIENCE}

$\psi_{i} \quad$ eigenfunction of the pre-ablation period

\section{Subscripts}

Av average potential

$i \quad$ index for dimensionless space

$j \quad$ index for dimensionless time

$m \quad$ melting of the materials

\section{Superscripts}

$\tilde{\theta} \quad$ integral transform preablation period

$\theta^{*} \quad$ dimensionless temperature ablation period

$\tilde{\theta}^{*} \quad$ integral transform ablation period

\section{INTRODUCTION}

The interaction between a particular material and the gas boundary layers is, in general, a complicated process. The least interaction occurs for those materials which melt and flow without evaporation; consequently this class of materials is firstly considered. Though melting materials are probably the least interesting from a practical point of view, an understanding of melting is fundamental to an overall understanding of the ablation problem.

Very little coupling exists between the flowing liquid layer and the external gas boundary layer, especially if the liquid velocity is small compared with the external gas velocity and the melting temperature is much less than the gas stagnation temperature. The heat transfer, shear and pressure distribution can then be regarded as known and equal to their values for a solid boundary at the melting temperature.

The ablation phenomenon is a process of thermal protection with several applications, mainly, in mechanical and aerospace engineering. Ablative thermal protection is applied using special materials (named ablative materials) externally on the surface of a structure in order to isolate it against thermal effects. The ablative phenomenon is a complex process involving phase changes with partial or total loss of material. Thus, the position of the boundary of the protection material not yet removed is initially unknown.

The diagrams shown in Figs. 1 and 2 illustrate the ablation phenomenon. In Fig. 1 the following processes are presented:
1. Convection heat transfer in the boundary layer that represents the main thermal load;

2. Radiation heat transfer;

3. Conduction heat transfer in the virgin material that should satisfy the temperature approach limits in the substructure or in the thermal shield on the structure;

4. Resin decomposition;

5. Fibers decomposition;

6. Flow of the produced gas through the residuals;

7. Retreat of the surface;

8. Radiation in the wall;

9. Shock in the boundary layer;

10. Combustion in the boundary layer.

Analytical, numerical, as well as analytical-numerical solutions have been found for this kind of problem. In a two-dimensional, geometry the method of Approximate Integral Balance, was presented by Hsiao and Chung (1983). The physical and mathematical models of the ablation process have been presented by Lacaze (1967). The solution of the diffusion problem with variable coefficients was studied by Cotta and Özisik, (1987). A generalized study of the ablative phenomenon was done by Adams (1959) and Sutton (1982). The using of the Generalized Integral Transform Technique (GITT), was presented by Diniz et al. (1993); Kurokawa et al. (2003); Gomes et al. (2004). They solved one-dimensional problems of heat diffusion for several geometries. Vallerani (1974) applied the Integral Method for problems of simple classes of ablation. The Classical Integral Transform Technique for linear problems was presented by Mikhailov and Özisik (1984). The GITT is a generalization of that technique for non-linear problems.

In the present work the one-dimensional analysis of the ablative process in a Finite Plate has been carried out using the Generalized Integral Transform Technique as an analytical tool for solution of the differential partial equation, considering the isolated internal side and the external side subject to a prescribed unsteady heat flow considering the radiation heat transfer effect. In the solution of the problem the value of interest is the temperature field in the material. 


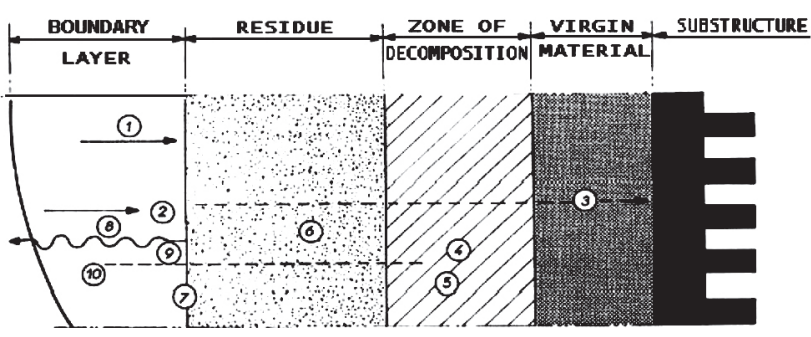

Figure 1. Illustration of ablation phenomenon

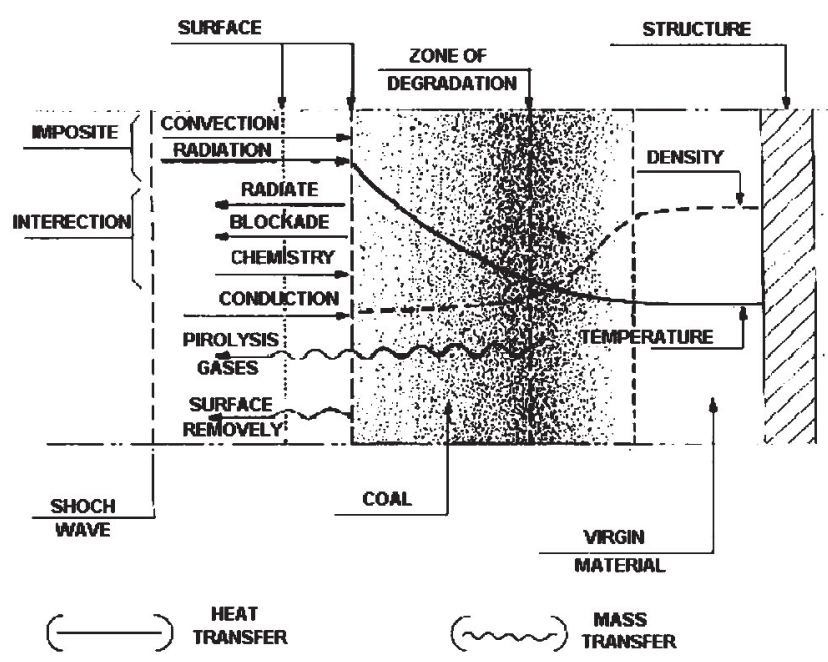

Figure 2. Physical representation of the ablation process involving the melting of the ablative material

\section{THEORETICAL ANALYSIS}

The radiation heat flux in a Finite Plate of finite thickness with constant physical properties and $\alpha$ (Absorptivity) $=\varepsilon$ (Emissivity) is considered. Initially, the plate is subjected to a temperature $T_{0}{ }^{*}$, its external surface is submitted to a prescribed unsteady heat flux and the internal surface is thermally insulated. This problem is solved in two steps: 1) the heating of the material until the phase change temperature is reached (preablation period), and 2) the ablation period that start at the heated surface through melting and continuous removal of the plate material (ablation period).

Figure 3 shows the plane geometry with a change of coordinate, from $X$ to $\eta$ coordinate, carried out to simplify the computational implementation. $Q(\tau)$ is the prescribed heat flux, $S(\mathrm{t})$ is the position of the ablative front in $X$ coordinate and $\eta_{\mathrm{b}}(\tau)$ (shaded area) represents the width of the ablative material that has not yet been consumed.

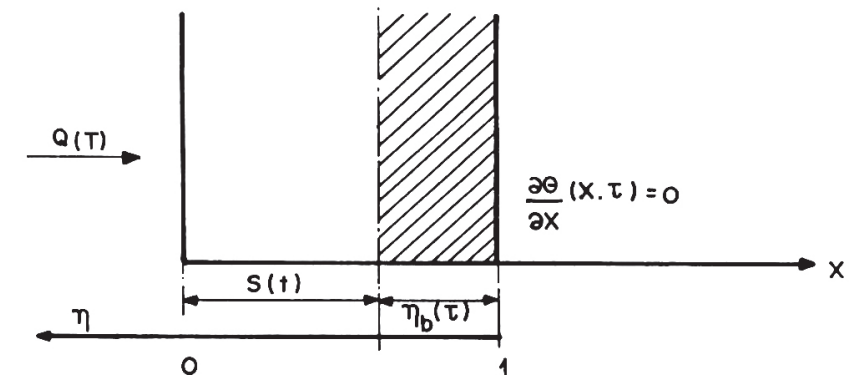

Figure 3. Geometry and coordinates system for ablation in a finite plate

\section{Description of the Problem for the Balance of Energy}

An ablating finite plate is considered an ablating finite plate which is fed into the plane $x^{*}=0$. Figure 4 shows a radiation heat flux $\left(q_{e}{ }^{*}\right)$ directly on the boundary. An asterisk indicates dimensional variables.

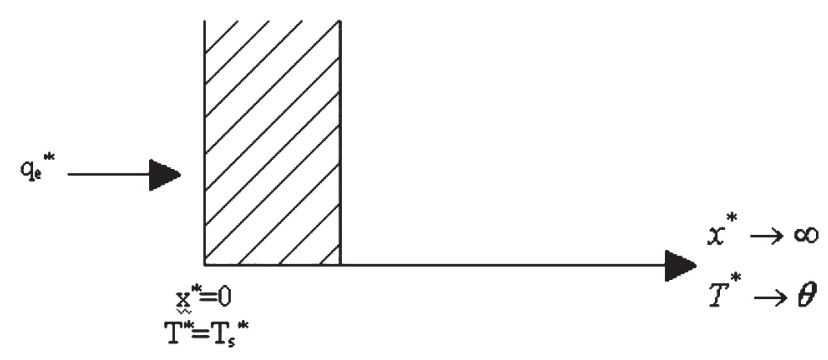

Figure 4. Schematic diagram of an ablating (and/or melting) surface. The melting rate is controlled by radiant energy input from an external source

\section{Preablation Period}

$$
\begin{gathered}
\frac{\partial \theta(X, \tau)}{\partial \tau}=\frac{\partial^{2} \theta(X, \tau)}{\partial X^{2}}+2 Q(\tau) E_{2}(x), \\
0<X<1,0<\tau<\tau_{m}
\end{gathered}
$$

with initial and boundary conditions:

$$
\begin{aligned}
& \theta(X, 0)=0,0 \leq X \leq 1 \\
& -\left.\frac{\partial \theta(X, \tau)}{\partial X}\right|_{X=0}=Q(\tau) \\
& \left.\frac{\partial \theta(X, \tau)}{\partial X}\right|_{X=1}=0, \tau>0
\end{aligned}
$$

and the function $\mathrm{E}_{2}(X)$ has been replaced by the Beer-Bouguer factor, $\exp (-X)$. 


\section{CIÊNCIA/SCIENCE}

Equations (1-4) define a standard radiation heat transfer problem, which is readily solved as a special case of the more general formulation presented by Mikhailov and Özisik (1984).

The following auxiliary problem with $\tau$-dependent quantities is chosen,

$$
\begin{aligned}
& \frac{\partial^{2} \psi_{i}(X)}{\partial X^{2}}+\mu_{i}^{2}(\tau) \psi_{i}(X)=0 \\
& 0<X<1,0<\tau<\tau_{m}
\end{aligned}
$$

subject to the boundary conditions:

$$
\frac{\partial \psi(X)}{\partial X}=0, X=0
$$

and

$$
\frac{\partial \psi(X)}{\partial X}=0, X=1
$$

which is readily solved to yield:

$$
\begin{aligned}
& \mu_{i}(\tau)=i \pi \\
& \psi i(X)=\cos \left[\mu_{i} X\right]
\end{aligned}
$$

Equation (5) allows the definition of the following integral transform pair Integral Transform:

$$
\tilde{\theta}(\tau)=\int_{X=0}^{X=1} \psi_{i}(X) \theta(X, \tau) d X
$$

Inversion Formula:

$$
\theta(X, \tau)=\sum_{i=1}^{\infty} \frac{\psi_{i}}{N_{i}} \tilde{\theta}(\tau)
$$

$\theta$ and $\theta$ are the terms that represent, respectively, the transformed integral and its inversion formula of the preablation period. $N_{\mathrm{i}}$ is the norm of the eigenfunctions, defined as

$$
N_{i}(\tau)=\int_{X=0}^{X=1} \psi_{i}^{2}(X) d X
$$

Using the GITT, it is possible to solve
Eq. (1) together with the boundary conditions, Eqs. (2)-(4). Thus, Eq. (1) can be solved to find the following temperature distribution:

$$
\theta(X, \tau)=\theta_{A V}(\tau)+\sum_{I=1}^{\infty} \frac{\psi_{i}(X)}{N_{i}} \tilde{\theta}_{i}(\tau)
$$

where, $\theta_{\mathrm{AV}}$ is the average potential, given by:

$$
\theta_{A V}=\int_{0}^{\tau} Q\left(\tau^{\prime}\right) d \tau
$$

The prescribed unsteady heat flux can be,

$$
Q(\tau)=A+B \tau+C \tau^{2}
$$

or

$$
Q(\tau)=D * e^{\tau / \tau_{r}}
$$

As shown by Hsiao and Chung (1983), using the polynomial flux and applying the technique in Eq. (13) yields:

$$
\theta_{A V}=F^{*}\left(A \tau+\frac{B \tau^{2}}{2}+\frac{C \tau^{3}}{3}\right)
$$

$$
\begin{gathered}
\theta(X, \tau)=F^{*}\left(A \tau+\frac{B \tau^{2}}{2}+\frac{C \tau^{3}}{3}\right)+ \\
-\sum_{i=1}^{\infty} \frac{\psi_{i}(X)}{N_{i}}\left\{\frac{E}{\mu_{i}^{2}}\left(A-\frac{B}{\mu_{i}^{2}}+\frac{2 C}{\mu_{i}^{4}}\right) e^{-\mu_{i}^{2} \tau}\right\}
\end{gathered}
$$

$$
\sum_{i=1}^{\infty} \frac{\psi_{i}(X)}{N_{i}}\left\{\frac{E}{\mu_{i}^{2}}\left[A+\left(B \tau-\frac{1}{\mu_{i}^{2}}\right)+C\left(\tau^{2}-\frac{2 \tau}{\mu_{i}^{2}}+\frac{2}{\mu_{i}^{4}}\right)\right]\right\}
$$

For the exponentials flux,

$$
\theta_{A V}=D * F * \tau_{r}\left(e^{\tau / \tau_{r}}-1\right)
$$

$$
\begin{aligned}
& \theta_{A V}=D^{*} F^{*} \tau_{r}\left(e^{\tau / \tau_{r}}-1\right) \\
& +\sum_{i=1}^{\infty} \frac{\psi_{i}(X)}{N_{i}}\left\{\frac{D^{*} E}{\mu_{i}^{2}+\frac{1}{\tau_{r}}}\left(e^{\tau / \tau_{r}}-e^{-\mu_{i}^{2} \tau}\right)\right\}
\end{aligned}
$$




\section{CIÊNCIA/SCIENCE}

Equations (10) and (11) represent the temperature distribution for $\tau<\tau_{M}\left(\tau_{M}\right.$ is the initial melting time of the material), for each adopted heat flux.

The value of $\tau_{M}$ is obtained from solving the transcendental Eq. (18) or (20), with

$$
\theta\left(0, \tau_{M}\right) \equiv 1
$$

Equations (18) and (20) represent the temperature distribution of the preablation period, for the considered flux.

\section{Ablation Period}

For the ablation period, Eq. (1) is again used, however, in this period the movement of the boundary begins, and the domain becomes $S(\tau)<X<1$, and correspondingly $\tau>\tau_{m}$. The initial and the boundary conditions are:

$$
\theta\left(X, \tau_{m}\right)=\theta_{m}(X), 0 \leq X \leq 1
$$

$$
\begin{gathered}
\theta(X, \tau)=1 \text { and } \frac{\partial \theta(X, \tau)}{\partial X}-v \frac{d S(\tau)}{d \tau}=Q(\tau), \\
\text { at } X=S(\tau) \\
\left.\frac{\partial \theta(X, \tau)}{\partial X}\right|_{x=1}=0, \tau>\tau_{m}
\end{gathered}
$$

where $\theta_{m}(X)$ is the temperature distribution within the slab when ablation starts, at $\tau=\tau_{m}$, obtained by solving Eq. (1), while the parameter $v$ in the heat balance for the ablating surface, Eq. (25), where $v$ is the inverse of the Stefan number and $\mathrm{S}(\tau)$ is the time-dependent position of the inward moving ablating boundary to be determined from Eq. (23).

For convenience, the boundary conditions should be homogenized, for this purpose the variable transformations were:

$$
\begin{aligned}
& \theta^{*}(X, \tau)=(X, \tau)-1 \\
& \eta=1-X
\end{aligned}
$$

$$
\eta_{b}(\tau)=1-S(\tau)
$$

After variable transformations, Eq. (1) becomes:

$$
\begin{gathered}
\frac{\partial \theta^{*}(\eta, \tau)}{\partial \tau}=\frac{\partial^{2} \theta^{*}(\eta, \tau)}{\partial X^{2}}+2 Q(\tau) E_{2}(X), \\
\tau>\tau_{M}, \quad 0<\eta<\eta_{b}(\tau)
\end{gathered}
$$

with initial and boundary conditions

$$
\theta^{*}\left(\eta, \tau_{m}\right)=\theta\left(\eta-1, \tau_{M}\right)-1
$$

$$
\begin{aligned}
& \left.\frac{\partial \theta^{*}(\eta, \tau)}{\partial \eta}\right|_{\eta=0}=0, \tau>\tau_{m} \\
& \theta^{*}(\eta, \tau)=0, \quad \tau \leq \tau_{m}, \quad \eta=\eta_{b}(\tau)
\end{aligned}
$$

and with the head balance at $\eta=\eta_{b}(\tau)$ given by:

$$
\frac{d \eta_{b}(\tau)}{d \tau}=\left.\frac{1}{v} \frac{\partial \theta^{*}(\eta, \tau)}{\partial \eta}\right|_{\eta=\eta_{b}(\tau)}-\frac{Q(\tau)}{v}, \tau>\tau_{m}
$$

and

$$
\eta_{b}\left(\tau_{m}\right)=1
$$

The following auxiliary problem for the situation of the ablation period with $\tau$-dependent quantities was chosen:

$$
\frac{\partial_{2} \phi_{i}(\eta)}{\partial \eta^{2}}+\mu_{i}^{2}(\tau) \psi_{i}(\eta), 0<\eta<\eta_{b}(\tau)
$$

$$
\left.\frac{\partial \phi_{i}(\eta)}{\partial \eta}\right|_{\eta=0)}=0 ; \phi_{i}\left[\eta_{b}(\tau)\right]=0
$$

which is readily solved to yield:

$$
\lambda_{i}(\tau)=\frac{(2 i-1) \pi}{2 \eta_{b}(\tau)}
$$

with, 


\section{CIÊNCIA/SCIENCE}

$$
\phi i(\eta)=\cos \left[\mu_{i}(\tau) \eta_{b}\right]
$$

Equation (34) allows the definition of the following integral transform pair:

Integral Transform:

$$
\tilde{\theta}_{i}^{*}(\tau)=\int_{0}^{\eta_{b}(\tau)} K i\left(\eta_{b}, \tau\right) \theta^{*}\left(\eta_{b}, \tau\right) d \eta
$$

Inversion Formula:

$$
\theta^{*}\left(\eta_{b}, \tau\right)=\sum_{i=1}^{\infty} K i\left(\eta_{b}, \tau\right) \tilde{\theta}_{i}^{*}(\tau)
$$

where $\tilde{\theta}_{i}^{*}$ and $\theta^{*}$ are the terms that represent, respectively, the transformed integral and the inversion formula of the ablation period, with the symmetric kernel defined as:

$$
k_{i}(\eta, \tau)=\frac{\psi_{i}(\eta, \tau)}{\left[N_{i}(\tau)\right]^{1 / 2}}
$$

and the normalization integral:

$$
N_{i}(\tau)=\int_{0}^{\eta_{b}} \psi_{i}^{2}(\eta) d \eta
$$

Applying the GITT technique, with Eq. (33) and $\tau=\tau_{M}$, it is possible to solve the ablation period, yielding:

$$
\begin{gathered}
\frac{d \tilde{\theta}_{i}^{*}(\tau)}{d \tau}+\lambda_{i}^{2}(\tau) \tilde{\theta}_{i}^{*}(\tau)+\sum_{j=1}^{\infty} A_{i j}(\tau) \tilde{\theta}_{j}^{*}(\tau) \\
-G Q(\tau) \cdot(-1)^{i+1} \cdot\left[\frac{(2 i-1) \pi}{(2 i-1)^{2} \pi^{2}+4}\right]=0 \\
\frac{\sqrt{2}}{2} \frac{\pi}{\left[\eta_{b}(\tau)\right]^{3 / 2}} \sum_{j=1}^{\infty}(2 j-1) \tilde{\theta}_{j}^{*}(\tau) \cdot(-1)^{j}-\frac{Q(\tau)}{v} \\
=\frac{d \eta_{b}(\tau)}{d \tau}
\end{gathered}
$$

Equations (42) and (43) form a system of infinite ordinary differential equations. To solve this system, it is necessary the substitution of that infinite system to a finite system of order $N$. The number of terms in the series, $N$, is set according to the desired accuracy. The truncated system of ordinary differential from Eqs. (42) and (43) is then:

$$
\begin{aligned}
& \frac{d \tilde{\theta}_{i}^{*}(\tau)}{d \tau}+\lambda_{i}^{2}(\tau) \tilde{\theta}_{i}^{*}(\tau)+\sum_{j=1}^{N} A_{i j}(\tau) \tilde{\theta}_{j}^{*}(\tau) \\
& -G Q(\tau) \cdot(-1)^{i+1} \cdot\left[\frac{(2 i-1) \pi}{(2 i-1)^{2} \pi^{2}+4}\right]=0 \\
& \frac{\sqrt{2}}{2} \frac{\pi}{\left[\eta_{b}(\tau)\right]^{3 / 2}} \sum_{j=1}^{N}(2 j-1) \tilde{\theta}_{j}^{*}(\tau) \cdot(-1)^{j}-\frac{Q(\tau)}{v} \\
& =\frac{d \eta_{b}(\tau)}{d \tau}
\end{aligned}
$$

Therefore the solution of the system of ordinary differential equations formed by Eqs. (44) and (45), gives the thickness and the rate of loss of the ablative material. These values have not yet been calculated up to this stage of the work. However, it has already been calculated the temperature distribution from Eq. (32) in the preablation period, considering the effect of radiation heat flux on the boundary.

\section{RESULTS AND DISCUSSION}

The distribution of temperature in the preablation period without radiation heat flux is presented in Fig. 5 and indicates a time of starting melting of the material of 0.196 , according to Diniz et al. (1993). The distribution of temperature in the preablation period with radiation heat flux at the boundary, presented in Fig. 6, shows that the temperature at the boundary reached the melting point faster than the case in which radiation is neglected. In fact, with radiation this time is only 0.0357 .

Figure 6 shows both the results without radiation and with radiation. Noticing the similar behavior between the temperature field, the effect of the radiation is clearly visible. So the material shall be consumed faster in the case of radiation.

In next step of this work the width of the ablative material and the velocity of the boundary will be calculated to compare them with results available in the literature. 


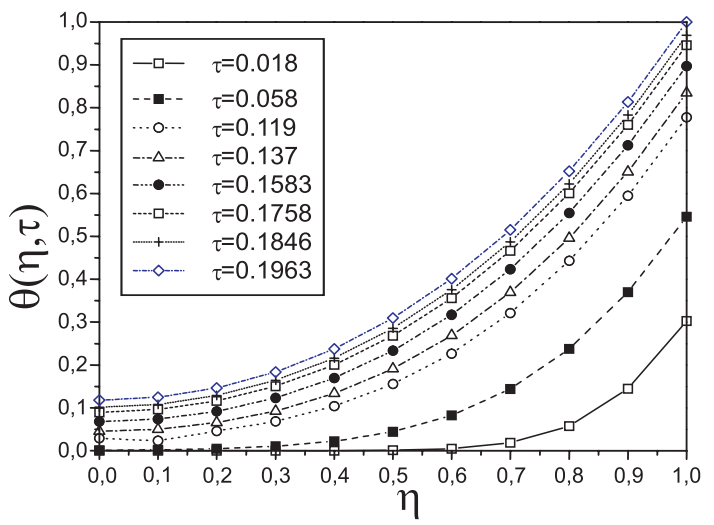

Figure 5. Distribution of temperature in the preablation period without radiation heat flux and $\mathrm{Q}(\tau)=2$

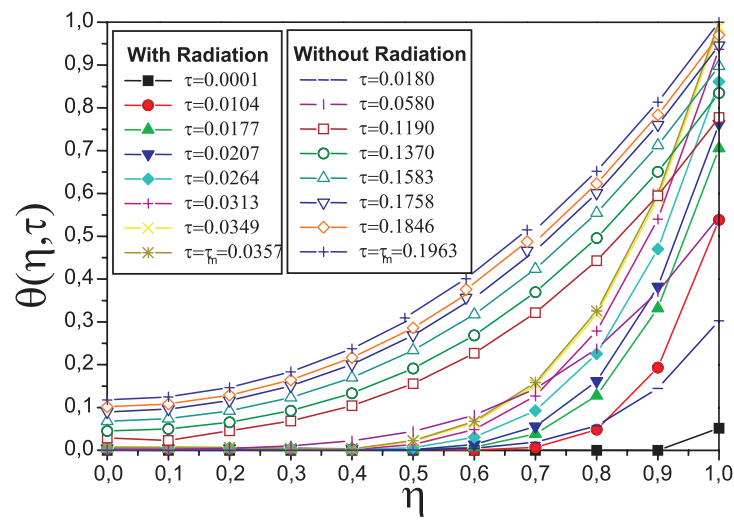

Figure 6. Comparison between the temperature field with and without radiation heat flux in the boundary and $\mathrm{Q}(\tau)=2$

\section{CONCLUSIONS}

The results of interest are the thickness $S(t)$ of the melted material, the velocity of ablation that can be calculated by $d S(t) / d t$ and the temperature field of the preablation and ablation periods. Here, just the temperature field of Pre-ablation has been calculated, to show the influence of radiation.

The thickness and the ablative speed are represented by the system of ordinary differential equations, Eqs. (37) and (38), which can be solved by numerical techniques in computational codes.

The temperature distribution with the presence of radiant energy, shown in Fig. 6, shows a fast increase in temperature with time, in comparison with the work without radiant energy, from Diniz et al. (1993), as shown in Fig. 6.

It is also possible to evaluate how shorter is the time for the radiant heat flux compared with the case where radiation is neglected, fact imposed for the high order of the exponent temperature in the analysis of the heat transfer for radiation. That fact is clearly observed by the form of the growth of the temperature distribution shown in Fig. 5. The effect of radiation is considered through the last term of the right hand side of Eq. (1), represented by the $E_{2}(X)$ term. This term is considered an exponential, because the phenomenon of the radiation is very fast.

The Generalized Integral Transform Technique has been applied with success in the present work, for the solution of the ablation with radiant energy problem in finite plate geometry. In the sequence of this work, the other results of interest, namely the depth and the velocity of ablation, will be calculated.

\section{REFERENCES}

Adams, M. C., 1959, Recent Advances in Ablation, ARS Journal, Avco - Everett Research Laboratory, Everett, Massachussets, USA.

Cotta, R. M., and Özisik, M. N., 1987, Diffusion Problems With General Time-Dependent Coefficients, Mechanical Sciences RBCM, Vol. 9, No. 4, pp. 269.

Diniz, A. J., Aparecido, J. B., and Cotta, R. M., 1993, Heat Conduction With Ablation in a Finite Slab, Integral Transforms in Computational Heat and Fluid Flow, Editor Cotta R. M., C. R. C. Press, c. 24, pp. 131-139 - Florida.

Gomes, F. A. A., Silva, J. B. C., and Diniz, A. J., 2004, Heat Transfer with Ablation in Cylindrical Bodies, In: 24th Congress of the International Council of the Aeronautical Sciences (ICAS), Yokohama, Japan.

Hsiao, J. S., and Chung, B. T. F., 1983, A Heat Balance Integral Approach for Two-Dimensional Heat Transfer in Solids With Ablation, AIAA-ASM- 22nd, Reno, Nevada.

Kurokawa, F. Y., Silva, J. B. C., and Diniz, A. J., 2003, Analytical/Numerical Hybrid Solution for One-Dimensional Ablation Problem, in Proceedings HT-2003-47174, ASME - Summer Heat Transfer Conference, Las Vegas, Nevada, USA.

Lacaze, H., 1967, La Protection Thermique Par Ablation, Doc-Air-Espace, No. 105.

Mikhailov, M. D., and Ozisik, M. N., 1984, Unified Analysis and Solutions of Heat and Mass Diffusion, John Willey \& Sons; New York.

Penner, S. S., and Olfe, D. B., 1968, Radiation and Reentry, A Volume in the Reentry Physics Series, Academic Press, New York and London, pp. 415-474.

Sutton, G. W., 1982, The Initial Development of Ablation Heat Protection, An Historical Perspective, AIAA - ASM - no. 4038 - Jan. - Fev., Everett, Massachussets, USA.

Vallerani, E., 1974, Integral Technique Solutions to a class of sinple Ablation Problems, Aeritalia Ricerca e Sviluppo Sistemi Spaziali, Settore Spazio, Torino, Itália. 